\title{
On the Equivalence of Matrix Differential Operators to Schrödinger Form
}

\author{
F. FINKEL $L^{\dagger *}$ and N. KAMRAN ${ }^{\ddagger \star}$ \\ †Departamento de Física Teórica II, Universidad Complutense, \\ 28040 Madrid, Spain \\ federico@ciruelo.fis.ucm.es \\ ${ }^{\ddagger}$ Department of Mathematics and Statistics, McGill University, \\ Montréal, Québec H3A 2K6, Canada \\ nkamran@math.mcgill.ca
}

\begin{abstract}
We prove a generalization to the case of $s \times s$ matrix linear differential operators of the classical theorem of E. Cotton giving necessary and sufficient conditions for equivalence of eigenvalue problems for scalar linear differential operators. The conditions for equivalence to a matrix Schrödinger operator are derived and formulated geometrically in terms of vanishing conditions on the curvature of a $g \ell(s, \mathbf{R})$-valued connection. These conditions are illustrated on a class of matrix differential operators of physical interest, arising by symmetry reduction from Dirac's equation for a spinor field minimally coupled with a cylindrically symmetric magnetic field.
\end{abstract}

\section{Introduction.}

Our purpose in this paper is to present a theorem which provides an explicit set of necessary and sufficient conditions for the local equivalence of eigenvalue problems associated to a general second-order linear $s \times s$ matrix differential operator in $\mathbf{R}^{n}$ and a matrix Schrödinger operator. We will consider equivalences which arise from the combination of local diffeomorphisms in $\mathbf{R}^{n}$ and conjugation of the matrix differential operator by nonsingular matrix-valued multiplication operators. In the scalar case $(s=1)$, this problem was solved on the line $(n=1)$ in [4] and in $n \geq 2$ dimensions in a classical paper of E. Cotton [1]. For matrix differential operators on the line, the local equivalence problem can also be solved explicitly [2].

The basic content of Cotton's theorem is that in order for a scalar linear second-order operator to be locally equivalent to a Schrödinger operator, a certain invariant 1-form constructed from coefficients of the differential operator using the Levi-Civitá connection of the metric determined by the principal symbol must be closed. This closure condition

Copyright (C) 1997 by Mathematical Ukraina Publisher. All rights of reproduction in form reserved.

* Supported by DGICYT grant PB92-097

* Supported by NSERC grant \#0GP0105490 
plays a very significant role in construction of quasi-exactly solvable Hamiltonians in $n \geq 2$ dimensions from a given normal form for a Lie algebra of first-order differential operators [3], [5]. The main goal of our note is to obtain a generalization of this closure condition in the matrix case. We shall see that the analogue of this closure condition in the matrix case is the vanishing of the curvature of a $g l(s, \mathbf{R})$-valued connection constructed from matrixvalued coefficients of the given operator. These zero curvature conditions are expected to play a significant role in construction of higher-dimensional quasi-exactly solvable matrix Schrödinger operators.

\section{Equivalence of linear differential operators.}

Our goal in this section is to define the natural notion of local equivalence for matrix linear differential operators in $\mathbf{R}^{n}$ which is best adapted to the study of eigenvalue problems. This is an obvious extension of earlier definitions formulated in the scalar case [3], [4].

Let $M$ and $\bar{M}$ denote open subsets of $\mathbf{R}^{n}$ with local coordinates given, respectively, by $x=\left(x^{1}, \cdots x^{n}\right)$ and $\bar{x}=\left(\bar{x}^{1}, \cdots \bar{x}^{n}\right)$. Consider on $M$ the $k$-th order linear $s \times s$ matrix differential operator.

$$
\mathbf{T}=\sum_{|I| \leq k} \mathbf{A}^{I} \partial_{I}
$$

where $I=\left(i_{1}, \cdots, i_{\ell}\right) \in \mathbf{N}^{\ell}$ denotes a multi-index,

$$
|I|=i_{1}+\cdots+i_{\ell}, \quad \partial_{I}=\frac{\partial^{|I|}}{\left(\partial x^{1}\right)^{i_{1}} \cdots\left(\partial x^{\ell}\right)^{i_{\ell}}},
$$

and the $\mathbf{A}^{I}$ are $s \times s$ matrices with entries in $C^{\infty}(M ; \mathbf{R})$. Likewise, on $\bar{M}$, consider an $s \times s$ matrix differential operator

$$
\overline{\mathbf{T}}=\sum_{|I| \leq k} \overline{\mathbf{A}}^{I} \bar{\partial}_{I} .
$$

We say that $\mathbf{T}$ and $\overline{\mathbf{T}}$ are equivalent if they can be transformed into each other by the combined effect of a change of variables and conjugation by a nonsingular $s \times s$ functional matrix, i.e., if there exists a local diffeomorphism $\varphi: M \rightarrow \bar{M}, \bar{x}=\varphi(x)$, and a $G L(s, \mathbf{R})$ valued function $\boldsymbol{\mu} \in C^{\infty}(M ; G L(s, \mathbf{R}))$ such that

$$
\left.\overline{\mathbf{T}}\left(\left.(\boldsymbol{\mu} \psi)\right|_{x=\varphi^{-1}(\bar{x})}\right)\right|_{\bar{x}=\varphi(x)}=\boldsymbol{\mu} \mathbf{T} \psi,
$$

for all $\psi \in C^{\infty}\left(M ; \mathbf{R}^{s}\right)$.

This notion of equivalence is particularly wellsuited to the study of eigenvalue problems since it preserves eigenvalues and induces a simple transformation law for eigenfunctions. Indeed, if $T$ and $\bar{T}$ are equivalent and if

$$
\mathbf{T} \psi=\lambda \psi,
$$

then we have

$$
\overline{\mathbf{T}} \bar{\psi}=\lambda \bar{\psi},
$$


where

$$
\bar{\psi}(\bar{x})=\left.(\mu \psi)\right|_{x=\varphi^{-1}(\bar{x})} .
$$

It is obviously an important problem to determine a workable set of necessary and sufficient conditions for two linear differential operators to be equivalent in the above sense. This is the problem we consider in Sections 2 and 3 in the case of 2nd-order operators.

\section{Equivalence of scalar 2nd-order operators - E. Cotton's theorem.}

The only case for which the equivalence problem defined in Section 1 has been solved explicitly is that of scalar 2nd-order operators in $n$ dimensions, corresponding to $k=2$ and $s=1$. For the case $n \geq 2$, this is a classical result, going back to E. Cotton [1]. For $n=1$, we refer to [4]. We now briefly recall the main step leading to Cotton's result, since they will serve as a guide for the matrix case.

Thus, we are given a scalar differential operator $T$ in $M$,

$$
T=\sum_{i, j=1}^{n} g^{i j} \frac{\partial^{2}}{\partial x^{i} \partial x^{j}}+\sum_{i=1}^{n} b^{i} \frac{\partial}{\partial x^{i}}+c,
$$

where $g^{i j}, b^{i}, 1 \leq i, \quad j \leq n$, and $c$ are real-valued $C^{\infty}$ functions in $M$. Likewise, we have an operator

$$
\bar{T}=\sum_{i, j=1}^{n} \bar{g}^{i j} \frac{\partial^{2}}{\partial \bar{x}^{i} \partial \bar{x}^{j}}+\sum_{i=1}^{n} \bar{b}^{i} \frac{\partial}{\partial \bar{x}^{i}}+\bar{c},
$$

with $C^{\infty}$ coefficients in $\bar{M}$.

It is easy to see that if $T$ and $\bar{T}$ are equivalent, then the $g^{i j}$ and $\bar{g}^{i j}$ transform like the components of a $(0,2)$ tensor and the quadratic forms associated to $g^{i j}(x)$ and $\bar{g}^{i j}(\varphi(x))$ must have the same rank and the same index. For applications to spectral problems in quantum mechanics, there is no loss of generality in assuming that these quadratic forms have a constant rank $n$ and index zero, meaning that they are everywhere positive-definite. We shall interpret $g^{i j}$ as contravariant components of a Riemannian metric $g_{i j}=\left(g^{i j}\right)^{-1}$ on $M$. We can thus rewrite $T$ in a manifestly covariant form by using the Levi-Civitá connection $\nabla_{i}$ of $g_{i j}$,

$$
T=\sum_{i, j=1}^{n} g^{i j}\left(\nabla_{i}-A_{i}\right)\left(\nabla_{j}-A_{j}\right)+U
$$

where the $A_{i}$ are components of a 1 -form and $U$ is a scalar defined by

$$
\begin{aligned}
& A^{i}=\sum_{j=1}^{n} g^{i j} A_{j}=-\frac{b^{i}}{2}+\frac{1}{2 \sqrt{g}} \sum_{j=1}^{n} \frac{\partial\left(\sqrt{g} g^{i j}\right)}{\partial x^{j}}, \\
& U=c+\sum_{i=1}^{n}\left(-A_{i} A^{i}+\frac{1}{\sqrt{g}} \frac{\partial}{\partial x^{i}}\left(\sqrt{g} A^{i}\right)\right),
\end{aligned}
$$




$$
g=\operatorname{det}\left(g_{i j}\right)
$$

We have a similar expression for $\bar{T}$ :

$$
\bar{T}=\sum_{i, j=1}^{n} \bar{g}^{i j}\left(\bar{\nabla}_{i}-\bar{A}_{i}\right)\left(\bar{\nabla}_{j}-\bar{A}_{j}\right)+\bar{U} .
$$

We shall denote the metrics and 1-forms associated to $T$ and $\bar{T}$ by

$$
\begin{aligned}
& d s^{2}=\sum_{i, j=1}^{n} g_{i j} d x^{i} d x^{j}, \quad d \bar{s}^{2}=\sum_{i, j=1}^{n} \bar{g}_{i j} d \bar{x}^{i} d \bar{x}^{j}, \\
& \mathcal{A}=\sum_{i=1}^{n} A_{i} d x^{i}, \quad \overline{\mathcal{A}}=\sum_{i=1}^{n} \bar{A}_{i} d \bar{x}^{i} .
\end{aligned}
$$

Note that since $s=1$, the factor $\mu$ appearing in (1.3) will just be a nonvanishing function $\mu \epsilon C^{\infty}\left(M ; \mathbf{R}^{*}\right)$. We have

$$
\begin{aligned}
& \mu^{-1} \sum_{i, j=1}^{n} g^{i j}\left(\nabla_{i}-A_{i}\right)\left(\nabla_{j}-A_{j}\right)= \\
& \quad=\sum_{i, j=1}^{n} g^{i j}\left(\nabla_{i}-A_{i}+\mu^{-1} \mu_{, i}\right)\left(\nabla_{j}-A_{j}+\mu^{-1} \mu_{, j}\right) .
\end{aligned}
$$

Using (2.10) and the tensoriality of $T$, we obtain:

Theorem 1 Necessary and sufficient conditions for $T$ and $\bar{T}$ given by (2.3) and (2.7) to be equivalent under a local diffeomorphism $\varphi: M \rightarrow \bar{M}, \bar{x}=\varphi(x)$, and conjugation by $\mu \in C^{\infty}\left(M ; \mathbf{R}^{*}\right)$ are given by

$$
\begin{aligned}
& \varphi^{*}\left(d \bar{s}^{2}\right)=d s^{2}, \quad \varphi^{*}(\overline{\mathcal{A}})=\mathcal{A}+\mu^{-1} d \mu, \\
& \varphi^{*}(\bar{U})=U .
\end{aligned}
$$

For applications to spectral problems in quantum mechanics, it is important to consider the case of Schrödinger operators

$$
H=-\frac{1}{2} \sum_{i, j=1}^{n} g^{i j} \nabla_{i} \nabla_{j}+V
$$

where $V \in C^{\infty}(M ; \mathbf{R})$ is a potential function.

Corollary 1 The differential operator $-\frac{1}{2} T$, where $T$ is given by (2.3), is equivalent to a Schrödinger operator $H$ if and only if

$$
d \mathcal{A}=0 .
$$

The closure condition (2.14) plays a crucial role in construction of quasi-exactly solvable potentials in $n \geq 2$ dimensions [3]. 


\section{Equivalence to matrix Schrödinger operators.}

Following the standard terminology, we define a matrix Schrödinger operator to be an $s \times s$ matrix differential operator of the form

$$
\mathbf{H}=-\frac{1}{2} \sum_{i, j=1}^{n} g^{i j} \mathbf{1}_{s} \nabla_{i} \nabla_{j}+\mathbf{V}
$$

where $g^{i j}$ 's are contravariant components of a Riemannian metric, $\nabla_{i}$ denotes the covariant derivative in the Levi-Civitá connection of that metric, $\mathbf{1}_{s}$ is the $s \times s$ identity matrix and $\mathbf{V}$ is an $s \times s$ symmetric (or Hermitian) matrix of functions of $x^{1}, \cdots, x^{n}$. Matrix Schrödinger operators arise naturally in Pauli's formulation of the nonrelativistic quantum mechanics for particles with spin. They also arise by symmetry reduction from the second-order matrix differential operator obtained by composing the Dirac operator with its formal adjoint, as shown by the following example.

Consider the Dirac equation

$$
\left(i \sum_{k=1}^{4} \gamma^{k}\left(\frac{\partial}{\partial x^{k}}+i e A_{k}\right)+m\right) \psi=0
$$

for a spinor field $\psi$ minimally coupled with a cylindrically symmetric magnetic field arising from a circular current in the $X Y$-plane. (We shall use the standard Weyl representation for the Dirac matrices $\left.\gamma^{k}, 1 \leq k \leq 4\right)$. Thus, there exists a gauge in which the vector potential is of the form

$$
\left(A_{i}\right)=\left(A_{t}, A_{r}, A_{\theta}, A_{z}\right)=\left(0,0, A_{\theta}(r, z), 0\right),
$$

where $(r, \theta, z)$ denote cylindrical coordinates.

In order to obtain a second-order operator, we act on the left of (3.2) with the formal adjoint of the Dirac operator given, of course, by

$$
-i \sum_{k=1}^{4} \gamma^{k}\left(\frac{\partial}{\partial x^{k}}+i e A_{k}\right)+m .
$$

This gives rise to a second-order equation for $\psi$, which decouples into two identical $2 \times 2$ matrix eigenvalue problems for a two-component spinor

$$
\psi=\left(\begin{array}{c}
\psi_{1} \\
\psi_{2}
\end{array}\right)
$$

We can the separate time and angular variables, in view of the symmetry of the problem. Thus, we let

$$
\left(\begin{array}{l}
\psi_{1} \\
\psi_{2}
\end{array}\right)=e^{-i E t}\left(\begin{array}{ll}
e^{i\left(j_{z}-\frac{1}{2}\right) \theta} & R_{1}(r, z) \\
e^{i\left(j_{z}+\frac{1}{2}\right) \theta} & R_{2}(r, z)
\end{array}\right)
$$

and we obtain the eigenvalue problem

$$
\mathbf{H}_{\mathrm{red}} \psi_{\mathrm{red}}=\lambda \psi_{\mathrm{red}}
$$


where

$$
\begin{aligned}
\mathbf{H}_{\mathrm{red}} & =\left(-\left(\frac{\partial^{2}}{\partial r^{2}}+\frac{\partial^{2}}{\partial z^{2}}\right)-\frac{1}{r} \frac{\partial}{\partial r}+e^{2} A_{\theta}^{2}\right) \mathbf{1}_{2}+\frac{1}{r^{2}}\left(\begin{array}{cc}
\left(j_{z}-\frac{1}{2}\right)^{2} & 0 \\
0 & \left(j_{z}+\frac{1}{2}\right)^{2}
\end{array}\right)- \\
& -2 e \frac{A_{\theta}}{r}\left(\begin{array}{cc}
j_{z}-\frac{1}{2} & 0 \\
0 & j_{z}+\frac{1}{2}
\end{array}\right)+e \frac{\partial A_{\theta}}{\partial z}\left(\begin{array}{ll}
0 & 1 \\
1 & 0
\end{array}\right) \\
\psi_{\text {red }} & =\left(\begin{array}{l}
R_{1} \\
R_{2}
\end{array}\right), \quad \lambda=E^{2}-m^{2} .
\end{aligned}
$$

By conjugating $\mathbf{H}_{\text {red }}$ with the matrix-valued multiplication operator given by

$$
\boldsymbol{\mu}=\operatorname{diag}\left(r^{-\frac{1}{2}}, r^{-\frac{1}{2}}\right),
$$

we arrive at the matrix differential operator $2 \mathbf{H}_{S}$, where $\mathbf{H}_{S}$ is the matrix Schrödinger operator given by

$$
\begin{aligned}
\mathbf{H}_{S} & =\left(-\frac{1}{2}\left(\frac{\partial^{2}}{\partial r^{2}}+\frac{\partial^{2}}{\partial z^{2}}\right)-e \frac{A_{\theta}}{r}+\frac{j_{z}^{2}}{2 r^{2}}\right) \mathbf{1}_{2}+ \\
& +\frac{e}{2} \frac{\partial A_{\theta}}{\partial z}\left(\begin{array}{ll}
0 & 1 \\
1 & 0
\end{array}\right)+\left(\frac{e}{2} \frac{A_{\theta}}{r}-\frac{1}{2} \frac{j_{z}}{r^{2}}\right)\left(\begin{array}{rr}
1 & 0 \\
0 & -1
\end{array}\right) .
\end{aligned}
$$

By construction, $\mathbf{H}_{S}$ will have the same eigenvalues as $\mathbf{H}_{\text {red }}$. This example provides a natural motivation for the study of the full equivalence problem to the matrix Schrödinger form (3.1), where the conjugating factor $\mu$ will now be replaced by a matrix-valued multiplication operator $\boldsymbol{\mu}$.

We observe that just as in the scalar case, form (3.1) for matrix Schrödinger operators is not invariant under conjugation by a nonsingular $s \times s$ matrix $\boldsymbol{\mu}$ of functions. In fact, the operator $\mathbf{W}$ defined by

$$
\mathbf{W}=\boldsymbol{\mu}^{-1} \mathbf{H} \boldsymbol{\mu},
$$

will be of the form

$$
\mathbf{W}=-\frac{1}{2} \sum_{i, j=1}^{n} g^{i j} \mathbf{1}_{s} \nabla_{i} \nabla_{j}+\sum_{i=1}^{n} \mathbf{B}^{i} \nabla_{i}+\mathbf{C},
$$

where $\mathbf{B}^{i}, 1 \leq i \leq n$, and $\mathbf{C}$ are $s \times s$ matrices. Therefore, it is natural to consider the equivalence problem for $s \times s$ matrix differential operators $\mathbf{T}$ and $\overline{\mathbf{T}}$ given by

$$
\begin{aligned}
& \mathbf{T}=\sum_{i, j=1}^{n} g^{i j} \mathbf{1}_{s} \frac{\partial^{2}}{\partial x^{i} \partial x^{j}}+\sum_{i=1}^{n} \mathbf{K}^{i} \frac{\partial}{\partial x^{i}}+\mathbf{L}, \\
& \overline{\mathbf{T}}=\sum_{i, j=1}^{n} \overline{\mathbf{G}}^{i j} \frac{\partial^{2}}{\partial \bar{x}^{i} \partial \bar{x}^{j}}+\sum_{i=1}^{n} \overline{\mathbf{K}}^{i} \frac{\partial}{\partial \bar{x}^{i}}+\overline{\mathbf{L}}
\end{aligned}
$$

where $g^{i j}$ 's are contravariant components of a Riemannian metric in $M$ and the remaining coefficients $\mathbf{K}^{i}, \mathbf{L}, \mathbf{G}^{i j}, \overline{\mathbf{K}}^{i}$, and $\overline{\mathbf{L}}$ are functions on $M$ and $\bar{M}$ taking values in the space of $s \times s$ matrices for all $1 \leq i, j \leq n$. 
Now, just as we did in the scalar case, we study the combined effect of a local diffeomorphism $\varphi: M \rightarrow \bar{M}, \bar{x}=\varphi(x)$, and conjugation by $\boldsymbol{\mu} \in C^{\infty}(M ; G L(s, \mathbf{R}))$ on the second-order terms in $\mathbf{T}$ and $\overline{\mathbf{T}}$. We obtain:

$$
\sum_{k, \ell=1}^{n} \overline{\mathbf{G}}^{k \ell} \frac{\partial x^{i}}{\partial \bar{x}^{k}} \frac{\partial x^{j}}{\partial \bar{x}^{\ell}}=g^{i j} \mathbf{1}_{s}, \quad 1 \leq i, j \leq n
$$

It should be noted that the matrix $\boldsymbol{\mu}$ by which we are conjugating does not appear in (3.16). From (3.16), it follows immediately that

$$
\overline{\mathbf{G}}^{k \ell}=\bar{g}^{k \ell} \mathbf{1}_{s}, \quad 1 \leq k, \ell \leq n,
$$

where $\bar{g}^{k \ell}$ are contravariant components of a Riemannian metric on $\bar{M}$, which by (3.16) is locally isometric to the metric defined on $M$ by $\left(g^{i j}\right)$.

We can therefore proceed in analogy with the scalar case and express $\mathbf{T}$ in covariant form as

$$
\mathbf{T}=\sum_{i, j=1}^{n} g^{i j} \mathbf{1}_{s}\left(\nabla_{i}-\mathbf{A}_{i}\right)\left(\nabla_{j}-\mathbf{A}_{j}\right)+\mathbf{U}
$$

where the $\mathbf{A}_{i}$ are the components of an $s \times s$ matrix- valued 1-form on $M$ and $\mathbf{U}$ an $s \times s$ matrix-valued function on $M$, defined by

$$
\begin{aligned}
& \mathbf{A}^{i}=\sum_{j=1}^{n} g^{i j} \mathbf{A}_{j}=-\frac{1}{2} \mathbf{K}^{i}+\frac{1}{2 \sqrt{g}} \sum_{j=1}^{n} \frac{\partial\left(\sqrt{g} g^{i j}\right)}{\partial x^{j}}, \\
& \mathbf{U}=\mathbf{L}+\sum_{i=1}^{n}\left(-\mathbf{A}_{i} \mathbf{A}^{i}+\frac{1}{\sqrt{g}} \frac{\partial}{\partial x^{i}}\left(\sqrt{g} \mathbf{A}^{i}\right)\right) .
\end{aligned}
$$

Similarly, we have on $\bar{M}$

$$
\overline{\mathbf{T}}=\sum_{i, j=1}^{n} \bar{g}^{i j} \mathbf{1}_{s}\left(\bar{\nabla}_{i}-\overline{\mathbf{A}}_{i}\right)\left(\bar{\nabla}_{j}-\overline{\mathbf{A}}_{j}\right)+\overline{\mathbf{U}} .
$$

If $\boldsymbol{\mu} \in C^{\infty}(M ; G L(s, \mathbf{R}))$, we have, in analogy with (2.10),

$$
\boldsymbol{\mu}^{-1} \mathbf{T} \boldsymbol{\mu}=\sum_{i, j=1}^{n} g^{i j} \mathbf{1}_{s}\left(\nabla_{i}-\boldsymbol{\mu}^{-1} \mathbf{A}_{i} \boldsymbol{\mu}+\boldsymbol{\mu}^{-1} \boldsymbol{\mu}_{, i}\right)\left(\nabla_{j}-\boldsymbol{\mu}^{-1} \mathbf{A}_{j} \boldsymbol{\mu}+\boldsymbol{\mu}^{-1} \boldsymbol{\mu}_{, j}\right)+\boldsymbol{\mu}^{-1} \mathbf{U}_{\boldsymbol{\mu}}
$$

From (3.22) and the tensoriality of $\mathbf{A}_{i}$, we deduce immediately that the $s \times s$ matrixvalued 1 -form $\mathcal{A}$, defined by

$$
\mathcal{A}=\sum_{i=1}^{n} \mathbf{A}_{i} d x^{i}
$$

transforms like the connection 1-form of a $g \ell(s, \mathbf{R})$-valued connection on $M$. 
Theorem 2 Necessary and sufficient conditions for $\mathbf{T}$ and $\overline{\mathbf{T}}$ given by (3.14) and (3.15) to be equivalent under a local diffeomorphism $\varphi: \bar{x}=\varphi(x)$ and conjugation by $\boldsymbol{\mu} \in$ $C^{\infty}(M ; G L(s, \mathbf{R}))$ are given by

(i) $\overline{\mathbf{G}}^{i j}=\bar{g}^{i j} \mathbf{1}_{s}$, where $\bar{g}^{i j}$ denote contravariant components of a Riemannian metric on $\bar{M}$.

(ii) $\varphi^{*}\left(d \bar{s}^{2}\right)=d s^{2}$, where

$$
d \bar{s}^{2}=\sum_{i, j=1}^{n} \bar{g}_{i j} d \bar{x}^{i} d \bar{x}^{j}, \quad d s^{2}=\sum_{i, j=1}^{n} g_{i j} d x^{i} d x^{j} .
$$

(iii) $\varphi^{*}(\overline{\mathcal{A}})=\boldsymbol{\mu}^{-1} \mathcal{A} \boldsymbol{\mu}-\boldsymbol{\mu}^{-1} d \boldsymbol{\mu}$.

(iv) $\varphi^{*}(\overline{\mathbf{U}})=\boldsymbol{\mu}^{-1} \mathbf{U} \boldsymbol{\mu}$.

It is now straightforward to obtain necessary and sufficient conditions for the matrix differential operator $\bar{T}$ given by (3.15) to be equivalent to a matrix Schrödinger operator $\mathbf{H}$ of the form (3.1).

Corollary 2 The matrix differential operator $-\frac{1}{2} \overline{\mathbf{T}}$, where $\overline{\mathbf{T}}$ is given by (3.15), will be equivalent to a matrix Schrödinger operator $\mathbf{H}$ of the form (3.1) if and only if:

(i) Conditions i) and ii) of Theorem 2 are satisfied

(ii) The $g \ell(s, \mathbf{R})$-valued connection defined by $\overline{\mathcal{A}}$ has zero curvature,

$$
d \overline{\mathcal{A}}+\overline{\mathcal{A}} \wedge \overline{\mathcal{A}}=0,
$$

or equivalently

$$
\overline{\mathcal{A}}_{i, j}-\overline{\mathcal{A}}_{j, i}-\left[\overline{\mathcal{A}}_{i}, \overline{\mathcal{A}}_{j}\right]=0
$$

(iii) The matrix potential $\mathbf{V}$ defined by

$$
\mathbf{V}(x)=-\left.\frac{1}{2} \overline{\boldsymbol{\mu}}^{-1} \overline{\mathbf{U}} \overline{\boldsymbol{\mu}}\right|_{\bar{x}=\varphi(x)},
$$

is Hermitian for some $\boldsymbol{\mu} \in C^{\infty}(\bar{M} ; G L(s, \mathbf{R}))$ solving

$$
\overline{\boldsymbol{\mu}}^{-1} \overline{\mathcal{A}} \overline{\boldsymbol{\mu}}-\overline{\boldsymbol{\mu}}^{-1} d \overline{\boldsymbol{\mu}}=0 .
$$

We finally remark that, as a consequence of Condition ii) of Theorem 2, the matrix differential operator $-\frac{1}{2} \overline{\mathbf{T}}$ will be equivalent to a matrix Schrödinger operator with a "flat" symbol

$$
\mathbf{H}=-\frac{1}{2} \sum_{i=1}^{n}\left(\frac{\partial}{\partial x^{i}}\right)^{2} \mathbf{1}_{s}+\mathbf{V}
$$

if and only if Condition i) of Theorem 2 and Conditions ii) and iii) of Corollary 2 are satisfied, and the Riemann-Christoffel curvature tensor of $\left(\bar{g}_{i j}\right)$ vanishes identically:

$$
\bar{R}_{j k \ell}^{i}=0 \text {. }
$$




\section{References}

[1] Cotton E., Sur les invariants différentiels de quelques équations aux dérivées partielles du second ordre, Ann. École Normale, 1900, V.17, 211-244.

[2] Finkel F., González-López A. and Rodríguez M.A., Quasiexactly solvable spin $\frac{1}{2}$ Schrödinger operators, UCM preprint.

[3] González-López A., Kamran N. and Olver P.J., New quasi-exactly solvable Hamiltonians in two dimensions, Commun. Math. Phys., 1994, V.159, 503-537.

[4] Kamran N. and Olver P.J., Equivalence of differential operators, SIAM J. Math. Anal., 1989, V.20, 1172-1185.

[5] Shifman M.A. and Turbiner A., Quantal problems with partial algebraization of the spectrum, Commun. Math. Phys., 1989, V.126, 347-365. 